\title{
Generation of Mid-IR Continuum Using Tellurite Microstructured Fiber
}

\author{
T. Delmonte, M.A. Watson, E.J. O’Driscoll \\ BAESystems ATC, Sowerby Building 20R, Filton, Bristol, BS34 9EQ, U.K.
}

\author{
X. Feng, T.M. Monro ${ }^{1}$, V. Finazzi, P. Petropoulos, J. Price, J. Baggett, W. Loh, D.J. Richardson \\ Optoelectronics Research Centre, University of Southampton, Southampton, SO17 1BJ, U.K.
}

D.P. Hand

Applied Optics and Photonics Group, Heriot-Watt University, Edinburgh, EH14 4AS, U.K.

We demonstrate the production of a well depleted mid-IR continuum with a spectral range between $\sim 0.9-2.5 \mu \mathrm{m}$ using a tellurite wagon wheel microstructured fiber and a pump source at $1.5 \mu \mathrm{m}$.

\section{Introduction}

Continuum generation has been observed in many different materials using a wide variety of pump regimes. However, there are limitations to the efficiency and wavelength regions available when using bulk material due to walk-off, material absorption etc. Optical fibers go a long way to solving conversion efficiency issues, with the small core diameter enabling a high intensity to be maintained over a very long length [1].

With the advent of microstructure fiber (MF) technology, extra design parameters allow the dispersion and confinement properties to be tailored for specific applications. For continuum generation, the light can be very tightly confined in a small core and the dispersion profile modified to generate nonlinear effects tailored to a specific source. However, continuum generation in silica glass is still limited to $2 \mu \mathrm{m}$ due to intrinsic absorption properties. As MF fabrication has matured, fibers made of more exotic glasses (where the material is transparent through to longer wavelengths) have been realised. These exotic glasses often have a higher inherent nonlinear co-efficient than silica, which coupled with the possibility of transmission past $2 \mu \mathrm{m}$ and very small core sizes have opened up efficient broadband continuum generation well into the mid-IR [2-4].

This paper demonstrates continuum in an extruded tellurite fibre up to $2.5 \mu \mathrm{m}$ which we believe is further into the mid IR regime than has been previously published in this type of microstructured material.

\section{Experimental Set-up}

The mid-IR source used for the experiment was an optical parametric oscillator (OPO), pumped using a tunable Coherent Mira femto-second laser $(\sim 800 \mathrm{~nm})$. The OPO was set up to be singly resonant and used a Stratophase PPLN crystal as the nonlinear medium. Average output power from the OPO was $\sim 160 \mathrm{~mW}_{\mathrm{av}}$ corresponding to a peak power of $\sim 11.7 \mathrm{~kW}_{\mathrm{pp}}$ at $1.56 \mu \mathrm{m}$ (equivalent to $2.1 \mathrm{~nJ}$ per pulse), FWHM $\sim 15 \mathrm{~nm}$.

The fiber itself was an extruded wagon wheel design (see inset in Figure 1), fabricated from sodium-zinctellurite glass $\left(75 \mathrm{TeO}_{2}-20 \mathrm{ZnO}-5 \mathrm{Na}_{2} \mathrm{O}\right.$, mol.\%). The fiber has an OD of $250 \mu \mathrm{m}$ with a central triangular core of diameter $2.7 \mu \mathrm{m}$ held in place by three struts, $7.4 \pm 0.2 \mu \mathrm{m}$ long and $180 \pm 20 \mathrm{~nm}$ thick. It is robustly single mode for wavelengths above $\sim 1 \mu \mathrm{m}$ and has been observed to support higher order modes at shorter wavelengths, while cut-off for single mode operation is between 0.635 and $1.047 \mu \mathrm{m}$ [5]. The nonlinearity $(\gamma)$ of the fiber at $1.55 \mu \mathrm{m}$ is $280(\mathrm{Wkm})^{-1}$, which is approximately 280 times greater than a standard silica fiber.

The output from the OPO source was coupled into the fiber using a standard 45 microscope objective, and taking into account the loss of the fiber $\left(2 \mathrm{dBm}^{-1}\right.$ at $\left.1550 \mathrm{~nm}\right)$, resulted in a slightly overfilled core and a coupling efficiency of $\sim 20 \%$. Alignment was carried out using am xyz-positioning stage and a thermopile detector to maximise output power. Radiation from the output was then coupled into a Jarrell-Ash monochromator using an array of $\mathrm{CaF}_{2}$ lenses to collimate and refocus the beam. After passing through the monochromator, the spectrum was measured using a nitrogen cooled InSb detector attached to an oscilloscope in roll mode. A system spectral curve using a Bentham CL2 white light source was also obtained to determine the combined response curves of the grating and detector. The resulting spectra takes into account the system spectral data. The lower half of the spectrum was measured using an Anritsu spectrum analyser, the two spectra were normalised to the OPO peak.

\footnotetext{
${ }^{1}$ Now at School of Chemistry and Physics, University of Adelaide, Australia
} 


\section{Experimental Results}

Using an approximate four wave mixing technique, the zero dispersion of the fiber was measured to be around $1.4 \mu \mathrm{m}$, (experimental error is estimated to be of the order $0.1 \mu \mathrm{m}$ ). Based on this value, we decided to pump slightly above this wavelength to obtain a broad spectra [6]. The pump wavelength of $1.56 \mu \mathrm{m}$ (a convenient source for many applications) was used, average output power from the fiber was $\sim 30 \mathrm{~mW}$ at a pump wavelength of $1.56 \mu \mathrm{m}$.

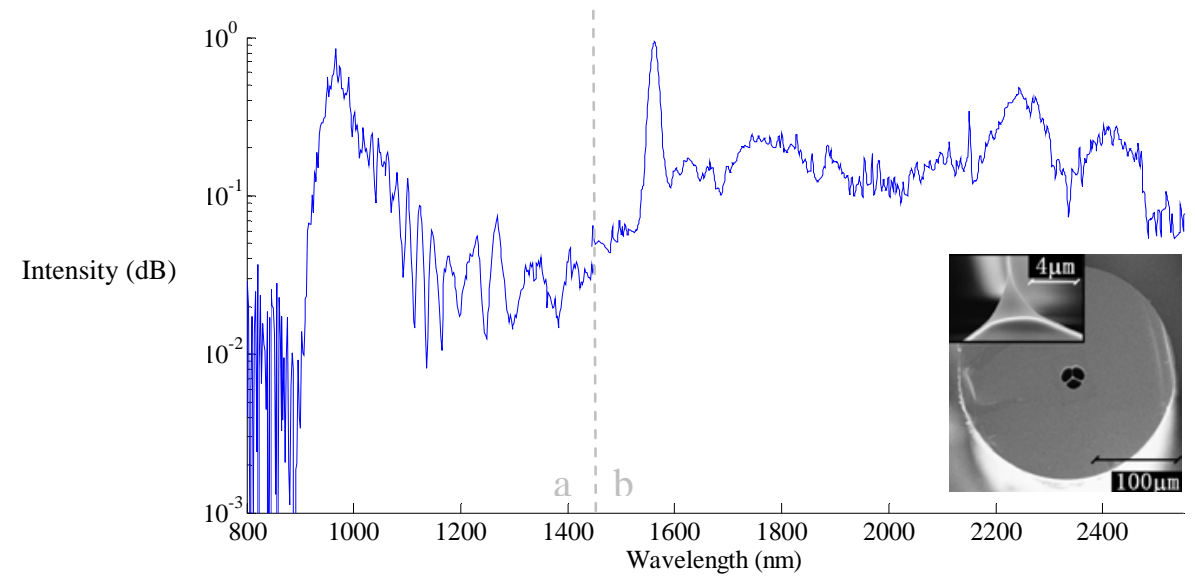

Figure 1: Output Spectra, a) is taken using a fiber coupled Anritsu spectrum analyser, and b) using a Jarrel-Ash monochromator (sensitive to above $2.6 \mu \mathrm{m}$ ), inset is an SEM of the fiber end-face, figure takes into account spectral response of detection equipment, no continuum signal detected above $2.5 \mu \mathrm{m}$

As can be seen in Figure 1, the spectrum had broadened significantly from the original OPO input. The broadening is attributed to soliton self frequency shift giving the fairly flat spectral shape. Below $1.4 \mu \mathrm{m}$, little power was observed until a large peak at $0.95 \mu \mathrm{m}$ with a magnitude similar to that of the pump peak. Some visible radiation was also observed in higher order modes at maximum input power. The generated spectrum seems to show that the actual zero dispersion point of the fibre is slightly shorter $(\sim 1.3 \mu \mathrm{m})$ than that measured.

\section{Conclusions}

We have demonstrated continuum well into the mid-IR region using an extruded tellurite wagon wheel fiber. The pump is well depleted, with broad flat generated spectra spanning from 1.4 to $2.5 \mu \mathrm{m}$. A large spectral feature is generated around $0.95 \mu \mathrm{m}$ with some visible radiation in higher order modes also observed. Currently the system is limited by the amount of power available for pumping the fiber, however if more power was available, results suggest that further spectral broadening past $2.5 \mu \mathrm{m}$ may be achieved.

\section{Acknowledgements}

Funding for this work had been provided by Selex Sensors and Airborne Systems. T. Delmonte is funded by the Engineering and Physical Sciences Research Council, U.K., through the Photonics Engineering Doctorate Centre based at Heriot-Watt University.

\section{References}

[1] The Supercontinuum Laser Source, Robert R. Alfano, ed. (Springer-Verlag 1989)

[2] T.M. Munro, Y.D. West, D.W. Hewak, N.G.R. Broderick, D.J., Richardson, “Chalcogenide holey fibres,” Electronic Letters, 36, 19982000, (2000)

[3] V.V. Kumar, A.K. George, W.H. Reeves, J.C. Knight, P,St.J. Russell, F.G. Omenetto, A.J. Taylor, “Extruded soft glass photonic crystal fiber for ultrabroad supercontinuum generation,” Optics Express, 10, 1520-1525, (2002)

[4] L.B. Shaw, P.A. Thielen, F.H. Kung, V.Q. Nquyen, J.S. Sanghera, I.D. Aggerwal, "IR Supercontinuum Generation in As-Se Photonic Crystal Fiber,” TuC5, ASSL 2005, Vienna

[5] X. Feng, T.M. Monro, V. Finazzi, R.C. Moore, K. Frampton, P. Petropoulos, D.J. Richardson, "Extruded singlemode, High-nonlinearity, tellurite glass holey fibre," Electronics Letters, 41, 835-837, (2005)

[6] A. Husakou, J. Herrmann, "Supercontinuum generation, four-wave mixing, and fission of higher-order solitons in photonic-crystal fibers,” J. Opt. Soc. Am. B., 19, 2181-2171, (2002) 